\title{
The usefulness of cytogenetic analysis in fine needle aspirates for the histologic subtyping of sarcomas
}

\author{
Scott E Kilpatrick ${ }^{1}$, Simon Bergman ${ }^{2}$, Mark J Pettenati ${ }^{3}$ and Margaret L Gulley ${ }^{1}$ \\ ${ }^{1}$ Department of Pathology and Laboratory Medicine, University of North Carolina, Chapel Hill, NC, USA; \\ ${ }^{2}$ Department of Pathology, Wake Forest University Baptist Medical Center, Winston-Salem, NC, USA and \\ ${ }^{3}$ Department of Pediatrics, Section of Medical Genetics, Wake Forest University Baptist Medical Center, \\ Winston-Salem, NC, USA
}

\begin{abstract}
Conventional cytogenetic analysis performed from open biopsy tissue samples may be a useful adjunct for the histologic subtyping of bone and soft tissue sarcomas. However, its diagnostic utility in fine needle aspiration biopsy (FNAB) specimens is unclear. We retrospectively reviewed 24 consecutive FNAB bone and soft tissue sarcoma specimens, procured from 1995 to 2003, in which aspirated material was obtained for cytogenetic analysis. The study sample included eight Ewing sarcomas, six synovial sarcomas, five rhabdomyosarcomas, two myxoid liposarcomas, and one each of myxoid chondrosarcoma, osteosarcoma, and atypical lipoma. Cytogenetic analysis confirmed the $t(X ; 18)$ in all six synovial sarcomas and the $t(11 ; 22)$ in three Ewing sarcomas. The $t(2 ; 13)$ was strongly suggested in one alveolar rhabdomyosarcoma. For two of these cases (both of which were synovial sarcomas), cytogenetic analysis was necessary for definitive diagnosis. While the positive cytogenetic results were supportive in the remainder, all were initially and accurately subtyped based on cytomorphology and/or immunohistochemistry. Cytogenetic analysis was noncontributory (eg no growth) in 14 sarcoma cases, but excluding the case of atypical lipoma, this did not preclude the rendering of an accurate diagnosis. Cytogenetic analysis can be performed on FNAB specimens from bone and soft tissue sarcomas and may be a useful diagnostic aid in difficult cases. However, when cell block material is available for immunohistochemistry, the majority of such cases are successfully subtyped.
\end{abstract}

Modern Pathology (2006) 19, 815-819. doi:10.1038/modpathol.3800598; published online 24 March 2006

Keywords: cytogenetic analysis; cytology; fine needle aspiration; sarcoma

The use of conventional cytogenetic analysis as an ancillary technique for the diagnosis of sarcomas is well documented in the surgical pathology literature. However, its reported usefulness in fine needle aspiration biopsy specimens (FNAB) is limited to rare series and isolated case reports, usually documented in clinical settings outside of the US. ${ }^{1-5}$ Not surprisingly, in our experience, many pathologists remain skeptical of the utility of FNAB for the diagnosis of sarcomas, and some appear unaware that such ancillary techniques as flow cytometry, karyotype, and fluorescence in situ hybridization

Correspondence: Dr SE Kilpatrick, MD, Pathologists Diagnostic Services, Forsyth Medical Center, 3333 Silas Creek Parkway, Winston-Salem, NC 27103, USA.

E-mail: sekilpatrick@novanthealth.org

This study was presented in part at the 92nd Annual Meeting of the United States and Canadian Academy of Pathology, Washington, DC, 22-28 March 2003.

Received 17 November 2005; revised 9 January 2006; accepted 10 January 2006; published online 24 March 2006
(FISH) may be successfully performed in routine clinical practice. We present herein our experience with the utility of FNAB and cytogenetic analysis as the initial diagnostic procedure of 24 patients. To our knowledge, this is the first report critically analyzing and evaluating the role, diagnostic accuracy, and utility of conventional cytogenetic analysis obtained from aspirates of a variety of bone and soft tissue sarcomas.

\section{Materials and methods}

We retrospectively reviewed 24 consecutive FNAB bone and soft tissue sarcoma specimens in which aspirated material was obtained for cytogenetic analysis from all 24 patients, none of whom had a previously established sarcoma diagnosis. All the material was reviewed by one (SEK) of us. The study sample included eight Ewing sarcomas; six synovial sarcomas; five rhabdomyosarcomas; two myxoid 
liposarcomas; and one each of myxoid chondrosarcoma, osteosarcoma, and atypical lipoma. All cytologic samples were obtained without general anesthesia using 23- and 25-gauge needles by the standard manual method at Wake Forest University Medical Center (WFU) (16 aspirates) and University of North Carolina Hospitals (UNC) (eight aspirates). Aspirated material was expelled onto glass slides and smeared using a second glass slide. Half of the smears were air dried and stained with Diff-Quik ${ }^{\circledR}$ (Fisher Scientific Biomedical Sciences Inc., Swedesboro, NJ, USA). An equal number of slides were immediately immersed in 95\% ethanol for staining by the Papanicolaou method. Immediately following the FNAB, a portion of the sample was screened utilizing the Diff-Quik ${ }^{\circledR}$ stain, and a preliminary interpretation rendered. Second or third FNAB were performed for additional material or studies (eg cell block and/or cytogenetics), when deemed potentially helpful for the diagnosis. Cell blocks were prepared from 23 FNAB specimens. For further details regarding our FNAB services and preparation of cell blocks, the reader is referred to a prior publication. ${ }^{1}$ When available and necessary (18 cases), sections of cell block material were examined immunocytochemically utilizing the avidin-biotinperoxidase complex and employing commercially available antibodies to the following antigens: S-100 protein (polyclonal 1/2; Dakopatts, Carpinteria, CA, USA); cytokeratin (monoclonal, AE1/AE3, 1/100 Boehringer Mannheim, Indianapolis, IN, USA), vimentin (monoclonal, 1/25; BioGenex, San Ramon, CA, USA); desmin (monoclonal, D33, 1/300, Dakopatts); muscle-specific actin (monoclonal, HHF-35, 1/25 000; Enzo Diagnostics, Farmingdale, NY, USA); and CD99 (monoclonal 013, 1/40; Signet, Dedham, MA, USA). Appropriate positive and negative controls were utilized throughout these procedures. Generally, we prefer a formalin-fixed block over cytologic smears for immunohistochemical evaluation, as adequate positive and negative histologic controls are readily available.

Specimens for cytogenetics were collected in RPMI 1640 tissue culture medium. FNABs were processed by cutting the tissue (if present) into minute fragments and incubating it in collagenase for $11 / 2 \mathrm{~h}$ with shaking at $36^{\circ} \mathrm{C}$ Two ml PC- 1 media ( $5 \%$ fetal calf; L-glutamine; Pen-Strep) was added to stop collagenase action. The supernatant was discarded and the cells were resuspended in $4 \mathrm{ml} \mathrm{PC-1}$ media and planted in two T-25 flasks. All cultures were incubated at $37^{\circ} \mathrm{C}$ in $\mathrm{CO}_{2} / \mathrm{N}_{2}$ incubators. After cell growth was established, media was removed, cells were rinsed with F-10 media (Life Technologies (Gibco), Grand Island, NY, USA) and then trypsinized $(10 \times \operatorname{trypsin} 0.05 \%$ and EDTA $)$ and plated onto 3-5 coverslips. Cells were incubated for 2-5 additional days. Colcemide was added for $2 \mathrm{~h}$, and the cultures were harvested and slides prepared using routine laboratory techniques. All metaphase cell preparations were GTG banded; 20 cells were examined and karyotypes were analyzed based on ISCN, 1995 nomenclature. ${ }^{6}$

\section{Results}

Those sarcomas in which cytogenetic results were successfully obtained are summarized in Table 1. Conversely, sarcomas in which FNAB failed to produce cytogenetic results are listed in Table 2. Cytogenetic analysis confirmed the $t(X ; 18)(p 11 ; q 11)$ in all six synovial sarcomas and the $t(11 ; 22)$ (q24;q12) in three (of eight) Ewing sarcomas. The $t(2 ; 13)$ was identified in one cell in one case (Case 10) of alveolar rhabdomyosarcoma. For two of the above karyotype 'positive' cases (both of which were synovial sarcomas), the karyotype result was crucial for definitive diagnosis, whereas karyotype was supportive of the histopathologic diagnosis in the remaining cases. In one of these cases (Case 7), the tumor cells were strongly and diffusely positive for CD99 and focally positive for cytokeratin (Figures 1-3). The differential diagnosis included Ewing sarcoma and synovial sarcoma. The results of cytogenetic analysis confirmed the $t(X ; 18)$, thus facilitating a diagnosis of synovial sarcoma (Figure 4). In the other case (Case 8), immunohistochemistry

Table 1 Clinicopathologic and cytogenetic features of the successful study cases

\begin{tabular}{|c|c|c|c|c|}
\hline Patient & Age/gender & Primary site & Cytologic diagnosis & Cytogenetic abnormality \\
\hline 1 & $5 / \mathrm{M}$ & R. femur & Ewing's sarcoma & $\mathrm{t}(11 ; 22)(\mathrm{q} 24 ; \mathrm{q} 12)$ \\
\hline 2 & $26 / \mathrm{M}$ & L. Buttock & Ewing's sarcoma & $\mathrm{t}(11 ; 22)(\mathrm{q} 24 ; \mathrm{q} 12)$ \\
\hline 3 & $6 / \mathrm{F}$ & R. Thigh & Ewing's sarcoma & $\mathrm{t}(11 ; 22)(\mathrm{q} 24 ; \mathrm{q} 12)$ \\
\hline 4 & $15 / F$ & R. Buttock & Synovial sarcoma & t(X;18)(p11;q11) \\
\hline 5 & $35 / \mathrm{M}$ & R. Thigh & Synovial sarcoma & $\mathrm{t}(\mathrm{X} ; 18)(\mathrm{p} 11 ; \mathrm{q} 11)$ \\
\hline 6 & $36 / \mathrm{M}$ & R. Ankle & Synovial sarcoma & $\mathrm{t}(\mathrm{X} ; 18)(\mathrm{p} 11 ; \mathrm{q} 11)$ \\
\hline 7 & $18 / \mathrm{M}$ & R. Buttock & Synovial sarcoma & $\mathrm{t}(\mathrm{X} ; 18)(\mathrm{p} 11 ; \mathrm{q} 11)$ \\
\hline 8 & $31 / \mathrm{M}$ & L. Thigh & Synovial sarcoma & $\mathrm{t}(\mathrm{X} ; 18)(\mathrm{p} 11 ; \mathrm{q} 11)$ \\
\hline 9 & 20/M & L. Foot & Synovial sarcoma & t(X;18)(p11;q11) \\
\hline 10 & $14 / \mathrm{M}$ & R. Neck & Alveolar RMS & $\mathrm{t}(2 ; 13)(\mathrm{q} 35 ; \mathrm{q} 14)^{\mathrm{a}}$ \\
\hline
\end{tabular}

$\mathrm{M}=$ male; $\mathrm{F}=$ female; $\mathrm{R}=$ right $\mathrm{L}=$ left RMS = rhabdomyosarcoma.

${ }^{\mathrm{a}}$ Documented in one cell. 
Table 2 Clinicopathologic features of the unsuccessful study cases

\begin{tabular}{|c|c|c|c|c|}
\hline Patient & Age/gender & Primary site & Cytologic diagnosis & Histologic diagnosis \\
\hline 1 & $16 / F$ & Shoulder & Ewing's sarcoma & Ewing's sarcoma \\
\hline 2 & $13 / \mathrm{M}$ & R. calcaneus & Ewing's sarcoma & Ewing's sarcoma \\
\hline 3 & $17 / \mathrm{M}$ & R. femur & Ewing's sarcoma & Ewing's sarcoma \\
\hline 4 & $7 / \mathrm{F}$ & L. foot & Ewing's sarcoma & Ewing's sarcoma \\
\hline 5 & $32 / \mathrm{M}$ & L. chest & Ewing's sarcoma & Ewing's sarcoma \\
\hline 6 & $11 / \mathrm{M}$ & L. neck & RMS & Alveolar RMS \\
\hline 7 & $14 / \mathrm{M}$ & L. cheek & RMS & Alveolar RMS \\
\hline 8 & $4 / \mathrm{F}$ & R. calf & RMS & Alveolar RMS \\
\hline 9 & 7/M & Abdomen & RMS & Embryonal RMS \\
\hline 10 & $37 / \mathrm{M}$ & L. buttock & MLS & MLS \\
\hline 11 & $29 / F$ & R. thigh & MLS & MLS \\
\hline 12 & $47 / \mathrm{M}$ & L. thigh & MCS & MCS \\
\hline 13 & $15 / F$ & L. femur & Osteosarcoma & Osteosarcoma \\
\hline 14 & $76 / \mathrm{M}$ & R. hand & Adipose tissue & Atypical lipoma \\
\hline
\end{tabular}

$\mathrm{M}=$ male; $\mathrm{F}=$ female; $\mathrm{R}=$ right $\mathrm{L}=$ left $\mathrm{RMS}=$ rhabdomyosarcoma; $\mathrm{MLS}=$ myxoid liposarcoma; $\mathrm{MCS}=$ myxoid chondrosarcoma

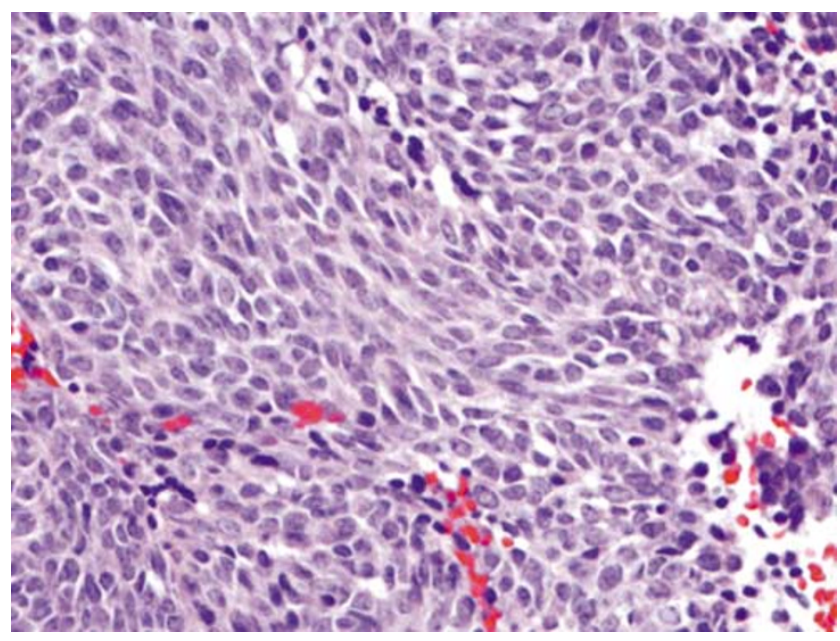

Figure 1 Case 7. Cell block showing a mostly uniform population of round to ovoid cells, suggestive of synovial sarcoma (Hematoxylin \& Eosin).

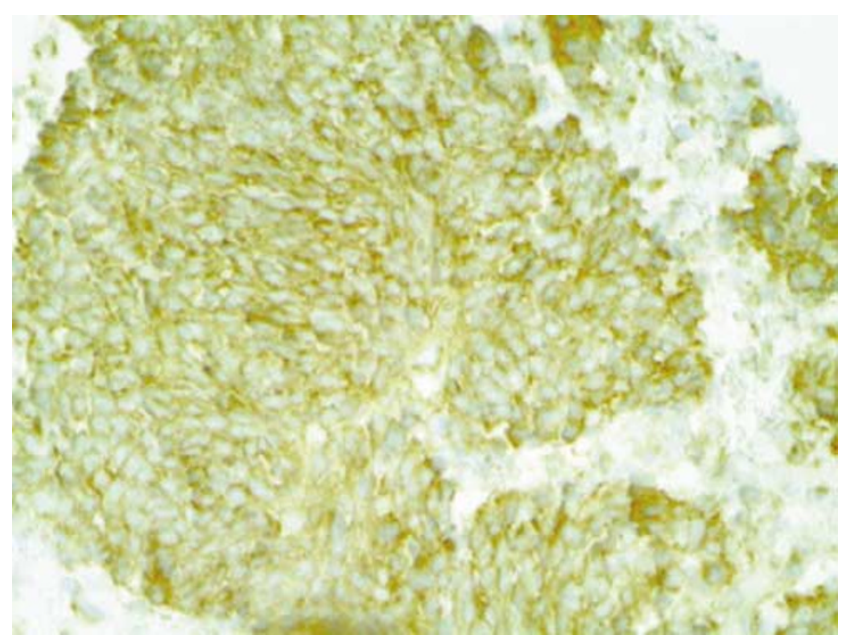

Figure 2 Case 7. Cell block. Tumor showing diffuse and strong membranous positivity for CD99.

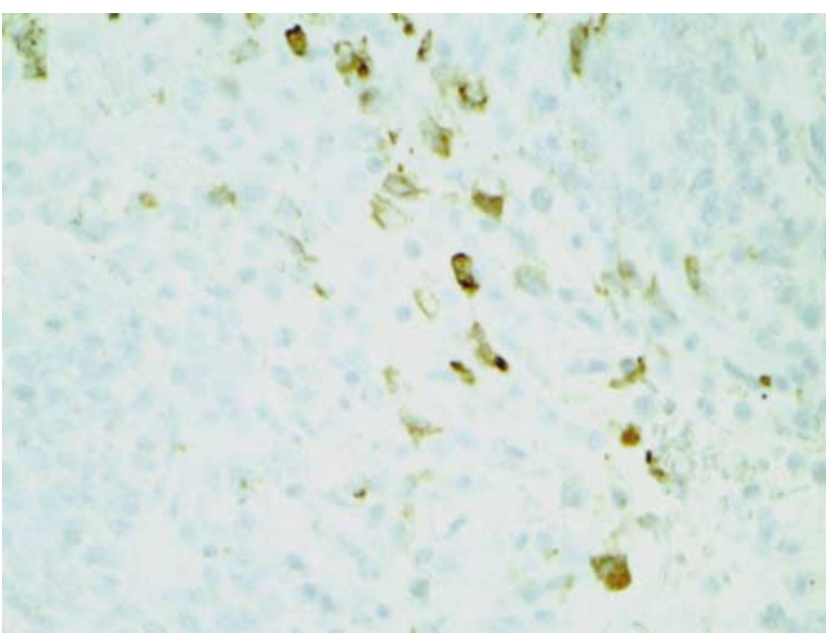

Figure 3 Case 7. Cell block. Tumor showing patchy and focal cytoplasmic positivity for cytokeratin.

could not be performed due to an inadequate cell block. While the positive cytogenetic results were merely supportive of histopathologic findings in the remainder of cases, all karyotypes were consistent with the classification, which had initially been based on cytomorphology and/or immunohistochemistry. Cytogenetic analysis was noncontributory (eg no growth) in 14 sarcoma cases but, excluding the case of atypical lipoma, did not preclude the rendering of an accurate diagnosis. Two other cases of alveolar rhabdomyosarcoma and one embryonal rhabdomyosarcoma produced no growth. Cytogenetic analysis was also attempted but was unsuccessful on two cases of myxoid liposarcoma and one case each of myxoid chondrosarcoma, osteosarcoma, and atypical lipoma. Immunohistochemistry was utilized for all but one (Case 8) of the cases listed in Table 1 and for Cases 1-9 in Table 2. 


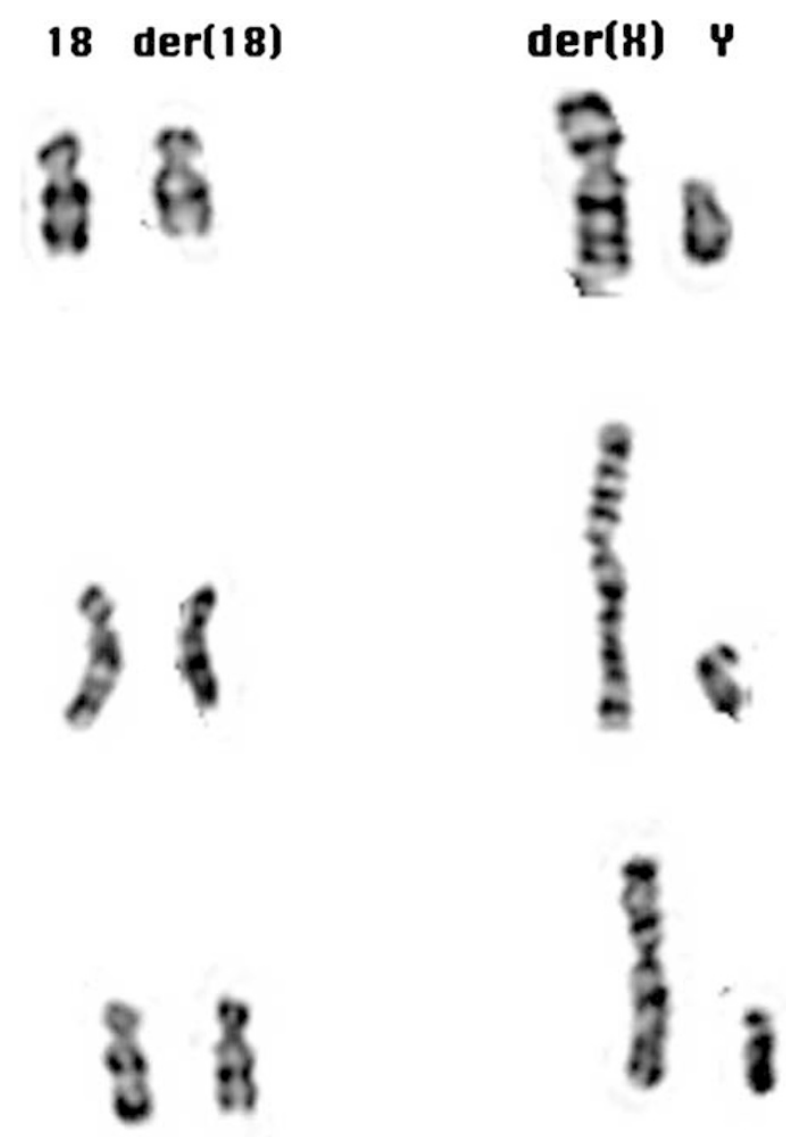

Figure 4 Case 7. Conventional cytogenetic analysis revealing the characteristic $\mathrm{t}(\mathrm{X} ; 18)$ of synovial sarcoma.

\section{Discussion}

This study illustrates the utility of cytogenetics in the routine work-up of sarcomas collected by FNAB. This is important since sarcomas are increasingly being recognized and classified by their genetic defects, and these defects are often visible by karyotype or FISH analysis. Furthermore, a cytogenetic defect serves as a tumor marker that might be helpful in staging or in measuring minimal residual disease. Novel therapies are now being explored that target the underlying genetic defects responsible for tumor cell growth, making it all the more important to identify these defects using genetic technologies.

In the past, a major argument against the use of FNAB for sarcomas has been its perceived unavailability of adequate material for necessary ancillary studies. ${ }^{7}$ However, the literature clearly demonstrates that one or more FNAB passes may obtain adequate material for immunocytochemistry (cell block), conventional cytogenetic analysis, flow cytometry and image analysis, and even research purposes, with informed consent of the patient. ${ }^{1,3,4,8,9}$ Other groups of investigators have successfully used polymerase chain reaction (PCR) amplification techniques on archival FNAB cytologic smears, confirming the EWS/FLI-1 fusion product of Ewing's sarcoma, the SYT/SSX fusion product of synovial sarcoma, and the detection of Herpesvirus-8 in cases of Kaposi's sarcoma. ${ }^{10-12}$ For the detection of the SYT/SSX fusion transcript by reverse transcriptase PCR, Inagaki et $a{ }^{11}$ reported a higher sensitivity among cytologic samples (91\%) compared to more traditional frozen biopsy materials $(75 \%)$. We have no experience with PCR and archival cytologic material. However, commercially available FISH probes can now be obtained for the detection of gene fusion products of most sarcomas with specific cytogenetic and gene fusion abnormalities. Recently, one of us (SB) successfully confirmed the SYT/SSX fusion gene transcript of synovial sarcoma on archival histologic and cytologic material, none of which represented the study cases. At the time of our study, these probes were not being utilized at either UNC or WFU for archival cytologic material.

Among bone and soft tissue tumors with diagnostically helpful and reproducible cytogenetic abnormalities (excluding one case of osteosarcoma), we were able to confirm such abnormalities using conventional cytogenetic analysis in 10 cases. As expected, we do not usually obtain aspirates for cytogenetic analysis unless our immediate cytologic assessment deems it potentially helpful for definitive diagnosis. For example, if the immediate assessment suggests a small round cell tumor (eg Ewing's sarcoma), we attempt to obtain material for cytogenetic analysis; conversely, if the differential diagnosis represents an adult pleomorphic sarcoma, we generally do not try to obtain such material, as these tumors generally do not have specific, reproducible cytogenetic abnormalities. In our experience, each ancillary study generally requires at least one additional (separate) FNAB pass (eg a cell block for immunocytochemistry necessitates one pass, cytogenetics studies require 1 pass, etc.). For the current cases, only one aspirate was performed and placed into cytogenetic media. Whether additional passes would have improved our yield is unclear and should be further investigated.

For the successfully cultured and karyotyped cases, there was a clear bias towards high-grade sarcomas. Higher-grade sarcomas typically yield a more proliferative cell line, likely lending itself to a higher success rate. Among high-grade sarcomas, we confirmed the $t(X ; 18)$ in all six cases of synovial sarcoma and the $\mathrm{t}(11 ; 22)$ in three (of 8) Ewing's sarcomas. For one alveolar rhabdomyosarcoma with characteristic small round blue cell morphology, a $t(2 ; 13)$ was found in one cell. In the literature, it appears that the best results for FNAB and cytogenetic analysis have been obtained in Ewing's sarcomas. ${ }^{3,4}$ We were not able to confirm cytogenetic abnormalities among any of our lowgrade sarcomas (eg myxoid liposarcoma, myxoid 
chondrosarcoma, and atypical lipoma), but perhaps newer molecular technologies will overcome the technical limitations of traditional karyotype in these cases.

It is no accident that bone and soft tissue tumors with relatively specific cytogenetic abnormalities also tend to have relatively specific immunohistochemical properties. For cases in which a cell block and immunohistochemical analysis were available and performed, accurate histologic subtyping occurred in all but one case (Case 7). This case represented a synovial sarcoma with cytomorphology that tended to favor this diagnosis (eg slightly spindled), but the immunohistochemistry was equivocal (eg strong and diffuse CD99 positivity and focal and patchy cytokeratin positivity). The differential diagnosis included Ewing's sarcoma. Cytogenetic analysis yielded the $\mathrm{t}(\mathrm{X} ; 18)$, which was crucial in making the diagnosis of synovial sarcoma. Cytogenetic analysis also helped resolve another case of synovial sarcoma (Case 8) in which a cell block was not obtained.

We do not believe that FNAB can reproducibly and reliably distinguish inadvertently sampled subcutaneous fat, lipoma, or well-differentiated lipoma-like liposarcoma, as all may contain areas of normal-appearing adipose tissue. ${ }^{1}$ Others have documented similar difficulties. ${ }^{13,14}$ For the above reasons, deeply seated soft tissue lesions that appear predominantly fatty by current imaging modalities are probably best evaluated by tissue biopsy. Our series included one case of atypical lipoma (welldifferentiated liposarcoma), involving the thenar eminence of the right hand that was not diagnosable by FNAB. The cytologic smears showed mostly normal-appearing adipose tissue; cytogenetic analysis was non-contributory.

In conclusion, conventional cytogenetic analysis may be successfully performed on FNAB specimens from bone and soft tissue sarcomas and is a useful diagnostic aid in difficult cases. Higher-grade sarcomas are more easily confirmed by cytogenetic analysis than lower-grade forms. Further studies are needed to address whether additional FNAB passes will increase the yield and success of cytogenetic analysis. Nevertheless, when cell block material is available for immunohistochemistry, the majority of sarcoma subtypes with specific cytogenetic profiles can be successfully classified with or without conventional cytogenetic analysis.

\section{References}

1 Kilpatrick SE, Cappellari JO, Bos GD, et al. Is fineneedle aspiration biopsy a practical alternative to open biopsy for the primary diagnosis of sarcoma? Experience with 140 patients. Am J Clin Pathol 2001;115:59-68.

2 Domanski HA, Carlen B, Mertens F, et al. Extraskeletal myxoid chondrosarcoma with neuroendocrine differentiation: a case report with fine-needle aspiration biopsy, histopathology, electron microscopy, and cytogenetics. Ultrastruct Pathol 2003;27:363-368.

3 Udayakumar AM, Sundareshan TS, Goud TM, et al. Cytogenetics characterization of Ewing tumors using fine needle aspiration samples. A 10-year experience and review of the literature. Cancer Genet Cytogenet 2001;127:42-48.

4 Willen H, Akerman M, Carlen B. Fine needle aspiration (FNA) in the diagnosis of soft tissue tumours; a review of 22 years experience. Cytopathology 1995;6: 236-247.

5 Udayakumar AM, Sundareshan TS, Appaji L, et al. Rhabdomyosarcoma: cytogenetics of five cases using fine-needle aspiration samples and review of the literature. Ann Genet 2002;45:33-37.

6 Mitelman F. International System for Human Cytogenetics Nomenclature. S Karger: Farmington, CT, 1995.

7 Association of Directors of Anatomic and Surgical Pathology. Recommendations for the reporting of soft tissue sarcomas. Am J Clin Pathol 1999;111:594-598.

8 Sapi Z, Antal I, Papai Z, et al. Diagnosis of soft tissue tumors by fine-needle aspiration with combined cytopathology and ancillary techniques. Diagn Cytopathol 2002;26:232-242.

9 Hughes JH, Caraway NP, Katz RL. Blastic variant of mantle-cell lymphoma: cytomorphologic, immunocytochemical, and molecular genetic features of tissue obtained by fine-needle aspiration biopsy. Diagn Cytopathol 1998;19:59-62.

10 Alkan S, Eltoum IA, Tabbara S, et al. Usefulness of molecular detection of human herpesvirus-8 in the diagnosis of Kaposi sarcoma by fine-needle aspiration. Am J Clin Pathol 1999;111:91-96.

11 Inagaki H, Murase T, Otsuka T, et al. Detection of SYTSSX fusion transcript in synovial sarcoma using archival cytologic specimens. Am J Clin Pathol 1999; 111:528-533.

12 Schlott T, Nagel H, Rushenberg I, et al. Reverse transcriptase polymerase chain reaction for detecting Ewing's sarcoma in archival fine-needle aspiration biopsies. Acta Cytol 1997;41:795-801.

13 Layfield LJ, Anders KH, Glasgow BJ, et al. Fine-needle aspiration cytology of primary soft-tissue lesions. Arch Pathol Lab Med 1986;110:420-424.

14 Ackerman M, Rydholm A. Surgery based on fine needle aspiration cytology. Acta Orthop Scand 1994; 65:69-70. 